\title{
Neue Hochdruck-Regeln für Zuckerkranke
}

\begin{abstract}
Seit Mitte Juni liegen die aktualisierten europäischen HypertonieLeitlinien vor. Sie enthalten auch Neuerungen bei Diabetikern.
\end{abstract}

Wichtige Neuerung ist ein einheitlicher Zielwert für die Blutdrucksenkung. Während zuvor differenzierte Zielwerte je nach Risikogruppe vorgegeben waren 140/90 mmHg bei niedrigem oder mäßigem Risiko, 130/80 mmHg für Hochrisikopatienten - gilt jetzt ein einheitlicher systolischer Zielwert von $140 \mathrm{mmHg}$. Diastolisch sollte eine Senkung unter $90 \mathrm{mmHg}$ erfolgen, speziell für Diabetiker werden Werte im Zielbereich zwischen 80 und $85 \mathrm{mmHg}$ empfohlen. Obligat ist eine antihypertensive Therapie bei systolischen Werten $\geq 160 \mathrm{mmHg}$, nachdrücklich empfohlen wird sie aber schon bei Werten $\geq 140 \mathrm{mmHg}$. Dazu, wie tief der systolische Blutdruck unter die Grenzen von $140 \mathrm{mmHg}$ fallen sollte, geben die Leitlinien mangels Evidenz keine Empfehlung.

\section{Kombi-Therapie ist quasi die Regel}

Da bei Diabetikern der Blutdruck oft schwierig einzustellen ist, ist bei dieser Risikogruppe eine antihypertensive Kombinationstherapie quasi die Regel. Wegen ihrer stärkeren Effekte auf die Proteinurie sollte stets ein ACE-Hemmer oder AT1-Rezeptorblocker in der Kombination enthalten sein. Von einer dualen RAS-Blockade mit ACE-Hemmer plus AT1-Rezeptorblocker wird abgeraten.

(ob)

\section{Ärzte Zeitung, 08.07.2013}

\section{"Wir wollen keine Linien mehr aufstellen und verantworten, dass alle Patienten pauschal auf diese oder jene Seite der Linie einsortiert werden."}

\section{Prof. Dr. David Matthews, Oxford}

- Patienten, für die Hypoglykämien schwerwiegende Folgen haben (gebrechliche Patienten/KHK-Patienten),

- Patienten mit langjähriger DiabetesErkrankung,

- multimorbide Patienten/Patienten mit manifesten kardiovaskulären Erkrankungen,

- geringe Lebenserwartung.

Als obligatorische Maßnahme zusätzlich zur Blutzuckersenkung wird in dem
Papier bei allen Patienten mit Typ-2Diabetes das Management kardiovaskulärer Risikofaktoren gefordert.

Dazu gehören insbesondere die Einstellung von Blutdruck und Cholesterinwerten sowie Maßnahmen zur Prävention einer diabetischen Nephropathie.

(DirkEinecke)

Ärzte Zeitung, 08.07.2013;

Grafik: Faramarz IB et al, Ann Intern Med 2011, 154(8): 554-559.
... ist bei Typ-2-Diabetikern mit komplexer Koronarerkrankung die Bypass-Operation das eindeutig bessere Verfahren zur Revaskularisation. Die FREEDOM-Studie belegt, dass damit im Vergleich zur Koronarintervention ( $\mathrm{PCl}$ ) und Stent-Implantation auch die Gesamtsterberate signifikant gesenkt wird.

...sind zwei größere Mahlzeiten (Frühstück und Mittagessen) für übergewichtige Typ-2-Diabetiker wohl günstiger als sechs kleinere inklusive Snacks. Nach Studiendaten einer Forschergruppe aus Prag hilft die "Zwei-Mahlzeiten“-Strategie bei der Gewichtsreduktion. Die Leberverfettung nahm stärker ab, die Verbesserung der Insulinsensitivität war ausgeprägter.

www.springermedizin.de/ Sommerakademie CME

In der e.Akademie von Springer Medizin können Sie bis zum 28. August 2013 kostenfrei punkten.

Zum Thema "Diabetes" finden Sie unter www.springermedizin.de/sommerakademie den Kurs „Therapieziele bei geriatrischen Patienten mit Diabetes mellitus - NoGos, Slow-Gos \& Go-Gos typgerecht behandeln". Um teilnehmen zu können, geben Sie bitte die Fortbildungs-Identifikationsnummer (FIN) in das entsprechende Feld ein.

\section{FIN: SOAK13MB}

kostenlos teilnehmen bis 28.08.2013

Viel Erfolg beim Punktesammeln!

Mit rund 1000 Kursen - viele davon als multimediale E-Learning-Formate - ist die e.Akademie Deutschlands erste Adresse für Online-CME-Fortbildung.

Die e.Akademie ist ein Modul von e.Med, dem Zugang zu allen digitalen Inhalten von Springer Medizin.

Testen Sie die e.Akademie 30 Tage lang kostenlos mit e.Med: www.springermedizin.de/eMed. 